\title{
A Positively Charged Surface Triggers Coagulation Activation Through Factor VII Activating Protease (FSAP).
}

\author{
Claudia Sperling ${ }^{1 *}$, Manfred F. Maitz ${ }^{1}$, Simona Grasso ${ }^{2}$, Carsten Werner ${ }^{1}$, Sandip M. Kanse ${ }^{2}$ \\ ${ }^{1}$ Institute Biofunctional Polymer Materials, Max Bergmann Center of Biomaterials, Leibniz- \\ Institut für Polymerforschung Dresden e.V., Dresden, Germany \\ ${ }^{2}$ Oslo University Hospital and University of Oslo, Oslo, Norway \\ * Corresponding author
}

Keywords: hemocompatibility; blood coagulation cascade; charged biomaterials; polyethylenimine; Factor seven activating protease; HABP2

\section{ABSTRACT (287 words)}

Contact between biomedical materials and blood often initiates undesirable pro-coagulant and pro-inflammatory processes. On negatively charged materials blood coagulation is known to be triggered through auto-activation of Factor XII, while activation on cationic surfaces follows a distinct and so far enigmatic mechanism. As Factor VII activating protease (FSAP), is known to be activated on positively and on negatively charged macromolecules in plasma, we have investigated its interaction with charged biomaterials and its consequences for coagulation. Several activation processes in blood and plasma were characterized after contact with material surfaces with varied charge. FSAP was found to be exclusively activated by the positively charged surfaces polyethylenimine (PEI) and poly-L-lysine (PLL), not by the negatively charged glass or self assembled monolayer with carboxyl group termination SAM-COOH) as well as uncharged (Teflon AF) surfaces. Whole blood incubation on PEI showed that this activation was concomitant with coagulation as determined by thrombin and fibrin formation which was high for glass (F1+2: $138 \mathrm{nM})$ and PEI (F1+2: $44 \mathrm{nM})$ but low for Teflon AF $(\mathrm{F} 1+2: 3.3 \mathrm{nM})$ and SAM COOH $(\mathrm{F} 1+2: 5.8 \mathrm{nM})$. Contact phase inhibitor diminished coagulation to background levels for all surfaces except PEI (F1+2: 43 to $25 \mathrm{nM}$; glass: 58 to $1.5 \mathrm{nM})$ indicating that coagulation activation is not dependent on FXII activation on the PEI surface. A decisive role of endogenous FSAP for coagulation however was confirmed with the use of FSAP inhibitory antibodies which showed no influence on Teflon AF, glass and SAM COOH but diminished F1+2 on PEI to less than $50 \%$. We propose that FSAP activation could be a novel mechanism of surfacedriven coagulation. An inhibition of this protease might improve hemocompatibility of cationic surfaces and therefore facilitate the application of polycationic surfaces in blood. 


\section{INTRODUCTION}

When blood contacts the artificial surface of medical devices or implants, enzymes of the intrinsic pathway of the coagulation cascade and activating mediators from adherent platelets and leukocytes induce blood coagulation. ${ }^{1}$ This applies to cardiovascular catheters, ${ }^{2}$ stents, artificial vessels as well as to hemodialysis ${ }^{3}$ and oxygenator membranes. ${ }^{4}$ Enormous effort was put in to modifying material surfaces to attain advanced hemocompatibility ${ }^{5}$ but the resulting medical products still are not fully satisfactory. In recent years there is an increased use of cationic materials for nanoparticles e.g. for intracellular gene or drug delivery (cancer treatment) or for polyelectrolyte multilayer (LBL), which in a denuded state, expose cationic surface groups. Additionally cationic surfaces were shown to have antimicrobial properties ${ }^{6}$ but their use is limited due to their procoagulant effect.

Patients that depend on these blood contacting devices are exposed to materials that activate several of the body's defense systems at once. Because of this they usually require a systemic anticoagulant therapy for the whole treatment period.

Initiation of the defense reactions of blood coagulation and inflammation results from protein adsorption processes which in turn are dictated by the chemical and physical surface characteristics of the exposed materials. ${ }^{7}$ Activating surface properties of materials that have been identified so far include hydrophobicity, negative surface charge and nucleophilic groups. Hydrophobic surfaces support fibrinogen adsorption and conformational change ${ }^{8}$ which correlates positively with platelet adhesion and activation. ${ }^{9,}{ }^{10}$ Negative surface charge induces FXII auto-activation to FXIIa ${ }^{11}$ which, as the initial step of the intrinsic pathway of the coagulation cascade, leads to thrombin activation and fibrin network formation. ${ }^{12}$ In isolated media this mechanism was shown to be not specific for anionic, hydrophilic surfaces. $^{13,} 14$ However in complex media (like blood plasma) competitive protein adsorption ${ }^{15}$ obviously prevents effective FXII activation on hydrophobic surfaces.

Another well explored mechanism is the activation of the complement system by nucleophilic groups (hydroxyl- or aminogroups) on the surface. They support complement fragment $\mathrm{C} 3$ hydrolysis and $\mathrm{C} 3 \mathrm{~b}$ binding, ${ }^{16-18}$ which initiates the alternative pathway of the complement cascade ${ }^{17,19}$ and results in the adhesion and activation of granulocytes and monocytes. ${ }^{8,11,20}$ All these reactions cannot be viewed as being separate but instead there is intense crosstalk between the different cascade systems and cells leading to a complex interaction of reactions at the blood-material interface. ${ }^{21}$ 
Less reliable knowledge exists on the activation of blood coagulation through positively charged surfaces. A strong activation of platelets ${ }^{22-24}$ or a repression of heparin activity through ionic interaction between the negatively charged molecule and the positive surface is thought to be involved. ${ }^{25}$ Another very different mechanism suggests that cationic surfaces lead to clot formation due to a direct interaction of the surface with fibrinogen. This was recently demonstrated to be the case with poly(amidoamine) PAMAM dendrimers. ${ }^{26}$ On the whole, none of these hypotheses has been substantiated enough to fully explain the documented procoagulant effects of positively charged surfaces. Detailed knowledge of the initiating procoagulant processes at the cationic surface might help to enhance the applicability of these materials. ${ }^{26,} 27$ Furthermore minimizing such undesired side effects would be highly desirable but requires a detailed understanding of the involved blood activation processes. ${ }^{1}$

FSAP which was also called hyaluronic acid binding protein 2 (HABP2) was first described in $1999^{28}$ for its capacity to directly activate $\mathrm{FVII}^{29}$ as well as urokinase-type plasminogen activator (u-PA) ${ }^{30}$ Even though biological relevance of the eponymic function of direct FVII activation has been disputed, ${ }^{31-33}$ FSAP is still considered to be an activator of the extrinsic pathway by interference with tissue factor pathway inhibitor (TFPI) ${ }^{33,34}$ and possibly with early forms of the prothrombinase complex. ${ }^{35,36}$

In a purified system, the zymogen form of FSAP is activated by negatively charged macromolecules like heparin, ${ }^{37,} 38$ nucleic acids and polyphosphates and also by positively charged molecules like histones. ${ }^{39,40}$ Crucially, only histones and nucleosomes can activate FSAP in the plasma. ${ }^{41}$ There is a transition of the single chain FSAP zymogen into a conformation supporting autoactivation by proteolysis of a peptide bond at the N-terminal region of the catalytic domain leading to the two chain form with a heavy and a light chain held together by a disulfide bond. ${ }^{42}$ This autoactivation process as well as the structure of FSAP has parallels to FXII. ${ }^{37-39}$ Like FXIIa, FSAP additionally has multiple activities related to coagulation, fibrinolysis and to inflammation. ${ }^{41,43,44}$

Considering these structural and functional similarities between the two coagulation factors XIIa and FSAP we hypothesize that FSAP is also involved in the surface-induced activation of coagulation. We have tested a set of defined polymeric materials with negative, positive or no surface charge in an in vitro blood incubation model with fresh human whole blood. Glass 
as well as self-assembled monolayers terminated with carboxylic groups (SAM-COOH) provide a negatively charged surface, polyethylenimine) (PEI) and poly-L-Lysine (PLL) showed cationic surface properties. PEI, a frequently used cationic material especially for gene transfer ${ }^{45}$ provides strong and stable positive surface charge consisting of primary, secondary and tertiary amine groups. As a branched polymer it provides surface chain flexibility. Yet contrary to some applications where PEI is added directly in solution our study uses PEI in an immobilized state. The other surface used was poly-L-lysine which exhibits only primary amine groups and less chain flexibility. PLL frequently is used to support cell adhesion and is known to provide stable positive charge. Teflon AF (teflon) did not provide ionizable surface groups and therefore can be regarded as a neutrally charged surface. Additionally hydrophobicity varied from the very hydrophilic surfaces glass and SAM-COOH to PEI and PLL with a moderate and Teflon AF with a strong hydrophobicity.

\section{MATERIALS AND METHODS}

\subsection{Surface preparation and characterization}

Materials with distinctly different, well characterized properties concerning surface charge and hydrophilicity (Table 1) were applied:

- PEI: polyethylenimine (PEI): positively charged groups of primary (app. $25 \%$ ), secondary (app. $50 \%$ ) and tertiary amines, soft surface, hydrogel-like thin network

- SAM-COOH $(\mathrm{COOH})$ : self-assembled monolayer with carboxylic groups: negative surface charge at physiologic $\mathrm{pH}^{46}$

- Glass: negative surface charge

- Teflon ${ }^{\text {TM }}$ AF (teflon): hydrophobic surface, no ionizable surface groups

- Poly-L-lysine (PLL): positively charged groups of primary amines, soft surface, hydrogel-like thin network

Table 1. Surface hydrophilicity and net surface charge. Dynamic contact angle (surface hydrophilicity) and isoelectric point (IEP, net surface charge) of tested surfaces: Dynamic contact angles of sessile water droplets $(8 \mu \mathrm{L})$ determined with an OCA 30 system (Dataphysics, Filderstadt, Germany), receding contact angles $<5^{\circ}$ correspond to no dewetting. IEP (isoelectric point) determined using streaming current measurement with $10 \mathrm{mM}$ $\mathrm{KCl}$ in an in-house developed set-up. ${ }^{47}$

\begin{tabular}{|l|l|c|}
\hline Material & Dynamic contact angle $\left.\boldsymbol{\theta}^{\boldsymbol{a}, \boldsymbol{r}} \boldsymbol{(}^{\circ}\right)$ & IEP \\
\hline Glass & $\boldsymbol{\theta}^{\boldsymbol{a}} 8 \pm 0^{\circ}, \boldsymbol{\theta}^{r}<5^{\circ}$ & 2 \\
\hline SAM-COOH & $\boldsymbol{\theta}^{a} 21 \pm 1.2^{\circ}, \boldsymbol{\theta}^{r}<5^{\circ}$ & 4.2 \\
\hline PEI & $\boldsymbol{\theta}^{\boldsymbol{a}} 67 \pm 0.5^{\circ}, \boldsymbol{\theta}^{r}<5^{\circ}$ & $>9.0^{48,49}$ \\
\hline Teflon AF & $\boldsymbol{\theta}^{\boldsymbol{a}} 120 \pm 0.5^{\circ}, \boldsymbol{\theta}^{r} 105^{\circ}$ & 4 \\
\hline PLL & $\boldsymbol{\theta}^{a} 62 \pm 3^{\circ}, \boldsymbol{\theta}^{r}<5^{\circ}$ & $>9.0$ \\
\hline
\end{tabular}


The net surface charge is zero at a $\mathrm{pH}$ that equals the IEP. It is negative for a $\mathrm{pH}$ above the IEP and positive for a $\mathrm{pH}$ below the IEP. Positively charged materials PEI and PLL show IEP of $>9$, while glass and SAM-COOH have an IEP of 2 and 4.2 respectively and are negatively charged in blood plasma ( $\mathrm{pH}$ 7.4). Teflon AF, while not providing ionizable surface groups, has an IEP of 4 independent of the nature and ionic strength of the electrolyte. Zimmermann et al. showed that this charging process at a nonpolar surface in simple electrolytes is primarily determined by the unsymmetrical adsorption of hydroxide and hydronium ions. ${ }^{50,51}$ This effect does not only apply to Teflon AF but generally to 'inert surfaces' - solid surfaces without ionizable surface groups. These ions are highly mobile and subject to competition processes if more complex fluids are contacting the surface. Therefore in a setting with physiologic salt concentration hydrophobic interactions are the dominant driving force for the interaction of proteins with the Teflon AF surface.

Glass (Borofloat glass disks $(25 \pm 1.1 \mathrm{~mm}$; Berliner Glas KG, Germany)) was cleaned thoroughly (mixture (1/1/5) of hydrogen peroxide (35\% by weight), ammonium hydroxide (29\%) and water at $70^{\circ} \mathrm{C}$ for $10 \mathrm{~min}$ )) right before application. Teflon AF (DuPont, Germany; solvent FC77 (1:5 (v/v)), an amorphous fluoroplastic was spincoated on glass discs, resulting layer thickness app $50 \mathrm{~nm}^{52}$ ). SAM-COOH was prepared as described ${ }^{11}: 11$ mercaptoundecanoic-acid (HS- $\left(\mathrm{CH}_{2}\right)_{10}-\mathrm{COOH}$; Sigma Aldrich, Germany) were adsorbed to glass disks coated with a $3 \mathrm{~nm}$ chromium layer followed by a $50 \mathrm{~nm}$ gold layer. Chemisorption was achieved from a $1 \mathrm{mM}$ thiol solution in absolute ethanol. PEI (M.W. 70,000; branched, $12.5 \mathrm{mg} / \mathrm{mL}$ in methanol: PolyScience Inc., USA) was used as a coating on surfaces and not in solution. It was spincoated on argon-plasma treated Teflon AF, and argon plasma treated again, approximate layer thickness: 5-10 nm. ${ }^{49,} 53$ Poly-L-lysine (PLL) (Sigma Aldrich, Germany; M.W. >30,000; $3 \mathrm{mg} / \mathrm{mL}$ in ethanol/MilliQ-water (1:1)) was spincoated on argon-plasma treated Teflon AF, and argon plasma treated again, resulting layer thickness app. $8 \mathrm{~nm}$.

\subsection{Whole blood/plasma incubation assay}

Blood incubation was performed in customized incubation chambers as reported before ${ }^{11,54}$ with freshly drawn venous blood pooled of two healthy voluntary ABO compatible donors, who denied use of any medication, especially non-steroidal anti-inflammatory drugs, during the previous ten days. The study was approved by the Ethics Committee of the Dresden 
University Hospital, Dresden, Germany. Informed consent was obtained from the donors prior to blood donation. Blood was anticoagulated (using heparin (1.7 IU/mL or as specified)) or hirudin where indicated (Refludan $1 \mu \mathrm{M}$ (Celgene Munich, Germany)) and filled immediately into pre-warmed autoclaved incubation chambers with the test materials and incubated at $37^{\circ} \mathrm{C}$ for 3 hours under permanent rotation. Blood incubation was repeated 2 times with $\mathrm{n}=6$ for all samples.

FSAP inhibitory antibody mab570 was added to the blood at $\mathrm{c}=100 \mu \mathrm{g} / \mathrm{mL}$ (concentration was proven to be sufficient for a full inhibition of FSAP in FXa generation assays on endothelial cells). ${ }^{34}$ Samples with addition of murine $\operatorname{IgG}$ isotype control antibody (100 $\mu \mathrm{g} / \mathrm{mL}$, Thermoscientific/Lifetechnologies, Germany) served as control. Incubation was performed in separate experiments with $n=2$ for each sample type except for $\mathrm{COOH}+$ mab570 with $\mathrm{n}=1$.

Corn trypsin inhibitor (CTI, Enzyme Research Laboratories, USA) to inhibit FXII activation was added before incubation at $c=10 \mathrm{U} / \mathrm{mL}$. Samples without CTI contained PBS as a control. Incubation with CTI was performed in 3 separate experiments with $n=9$ for samples without inhibitor, $\mathrm{n}=1$ for Teflon $\mathrm{AF}+\mathrm{CTI}$ and $\mathrm{COOH}+\mathrm{CTI}$ and $\mathrm{n}=7$ for glass $+\mathrm{CTI}$ and PEI+CTI.

Blood $\mathrm{pH}$ and blood gases were determined before and after incubation. The $\mathrm{pH}$ value was stable during the incubation at $\mathrm{pH} 7.4$ for all samples, also gas values remained stable.

Samples for blood cell count and flow cytometry were analysed immediately after surface incubation. Samples for the prothrombin fragment $1+2(\mathrm{~F} 1+2)$ and thrombin-antithrombincomplex (Enzygnost F1+2, TAT-micro; Siemens Healthcare, Germany), free complement fragment C5a (DRG Instruments GmbH, Germany), platelet factor 4, D-Dimer and Fibrinopeptid-A (Zymutest PF4, DDimer, FPA; Hyphen BioMed, France) were taken.

Red and white blood cells and platelets were counted in EDTA anticoagulated blood using the automated cell counter Coulter Act.diff.

For flow cytometry cells were stained with VioBlue labelled anti CD11b (Miltenyi Biotec $\mathrm{GmbH}$, Germany) for $30 \mathrm{~min}, 0.1 \%$ (wt/vl) $\mathrm{NaN}_{3}$ was added. ${ }^{55}$ To detect platelet-granulocyte conjugates additional staining with FITC labeled anti CD41a (BD Biosciences, Germany). The median intensity of the CD11b signal of the granulocyte population was quantified. The formation of platelet-granulocyte conjugates was determined as the rate of CD41a positive granulocytes. 
For analysis of surface complement or for scanning electron microscopy (SEM) sample surfaces were washed with PBS and further prepared as described before. ${ }^{11}$

\subsection{Platelet adhesion to surfaces}

To determine the direct interaction of platelets with surfaces without interference of other cells and coagulation processes we additionally determined the adhesion of blood platelets from citrated platelet rich plasma (PRP) on the surfaces. This was achieved as described before by measuring the amount of lactate dehydrogenase (LDH) release after cell lysis with Triton-X (1\% vol/vol). ${ }^{11}$

\subsection{Complement adsorption to surfaces}

Complement adsorption was detected as described before. ${ }^{11}$ Additionally to polyclonal antiC3c, (1:500, DakoCytomation, Denmark) monoclonal anti-C4d (clone 12D11, 1:50, Hycult biotech, Netherlands) was used for the detection of adsorbed complement fragments.

\subsection{Contact activation (activity of FXIIa / kallikrein)}

Activated coagulation factor XII (FXIIa) together with kallikrein activity was determined as described before using a chromogenic substrate solution $(0.3 \mathrm{mM} \mathrm{S} 2302$, Chromogenix, USA). ${ }^{11}$

\subsection{Determination of FSAP concentration and activation}

Whole blood or $300 \mu \mathrm{L}$ of hirudin-plasma was incubated on the surfaces for indicated times $(1,20,60,120 \mathrm{~min})$ at $37^{\circ} \mathrm{C}$ under constant agitation and then concentration and activation of FSAP was detected.

FSAP- $\alpha 2$-antiplasmin complexes were measured as described before ${ }^{44}$ except that standards were prepared with plasma FSAP activated with histones $(50 \mu \mathrm{g} / \mathrm{mL})$ for $1 \mathrm{~h}$ at $37^{\circ} \mathrm{C}$. FSAP antigen and activity were measured by ELISA and chromogenic substrate conversion after immunofixation, respectively, as previously described. ${ }^{56}$

\subsection{SDS PAGE and Western blot analyses}

Plasma or surface extracts were separated by 10 or $12 \%$ SDS-PAGE under reducing (10\% mercaptoethanol) or non-reducing conditions. Proteins were transferred onto PVDF 
membrane (Millipore, USA) and probed with primary antibodies. Bound antibodies were detected using horseradish peroxidase conjugated secondary antibodies (Dako, Denmark) and the enhanced chemiluminescence detection system (Amersham-Pharmacia, GE Healthcare, Germany). Blots were probed with rabbit polyclonal anti FSAP antibody (kindly donated by Michael Etscheid (Langen, Germany)).

\subsection{Statistical Analysis}

All surfaces were tested in triplicate, the experiments were repeated twice accounting to $n=6$ for most of the results (if not indicated otherwise). Graphs present mean +/- standard deviation. Statistical evaluation was performed by one-way ANOVA and a subsequent Tukey's multiple comparison test if not indicated otherwise (GraphPad Software version 6, LaJolla, CA). Significance was defined as $p<0.05$.

\section{RESULTS}

All the surfaces included in this study were characterized with respect to surface hydrophilicity and charge before their use for the blood and plasma incubation. These results are presented in the Materials and Methods section and in Table 1.

\subsection{Activation of coagulation and fibrinolysis}

Blood incubation was performed in customized incubation chambers with fresh whole human blood anticoagulated with a low dose of heparin (to weaken pro-coagulant processes to better differentiate between strong and weak activating material). Scanning electron microscopy of the tested surfaces incubated with blood revealed a strong clotting response on negatively charged glass and PEI (Figure 1). No fibrin deposition was seen on hydrophobic, uncharged Teflon AF or hydrophilic, negatively charged SAM-COOH (Figure 1). SAM-COOH showed prominent adhesion and spreading of granulocytes, which was much more than on the other surfaces. Strong platelet adhesion with distinct spreading was seen on Teflon AF; but only few platelets were observed on SAM-COOH. Platelet adhesion from whole blood on PEI and glass was so strong that it could not be analyzed accurately. To overcome this problem platelet adhesion was additionally analysed incubating surfaces with platelet rich plasma (PRP). The highest density of platelets was seen on Teflon AF (Figure S1). PEI showed approximately $80 \%$, glass and SAM-COOH $20 \%$ of adherent cells compared to Teflon AF. This scales well with surface hydrophobicity, an effect that we showed before but that has no direct correlation to the procoagulant capacity. ${ }^{11}$ 

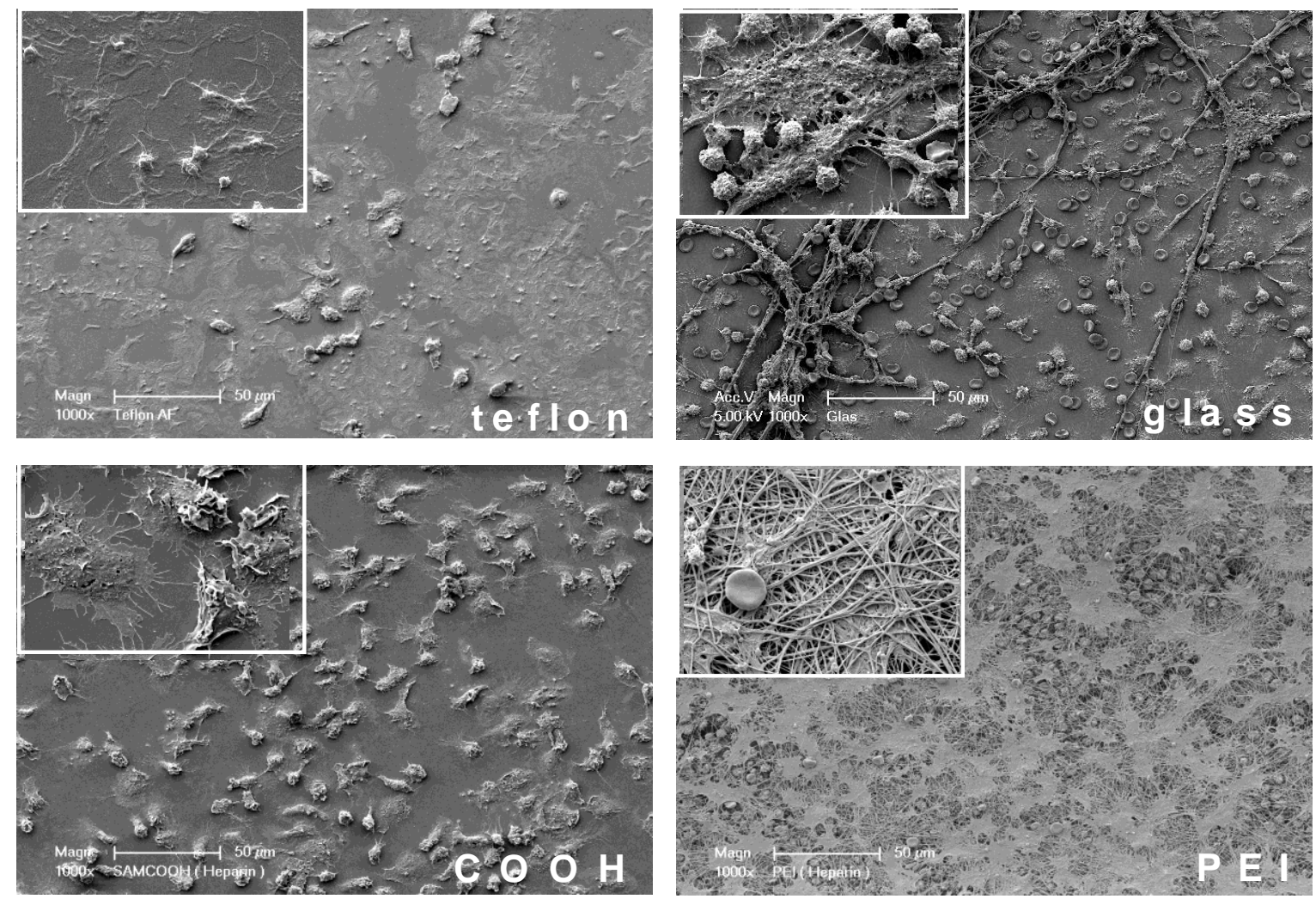

Figure 1. Surface adherent blood cells and fibrin deposition. In vitro whole blood incubation (3 hours at $37^{\circ} \mathrm{C}, 1.7 \mathrm{IU} / \mathrm{ml}$ heparin) Magnification 1000x, inserts: magnification 4000x; ESEM XL 30 FEG, FEI-Philips, Eindhoven, The Netherlands. Strong platelet adhesion with distinct spreading on hydrophobic Teflon AF (teflon); only few platelets on SAM-COOH $(\mathrm{COOH})$. This surface is characterized through the adhesion and spreading of granulocytes. Glass and PEI showed a strong formation of fibrin mesh - being clearly visible on PEI while on glass massive clots were lost during the rinsing / fixation process. Thus, both the glass and PEI surface show a strong activation of coagulation.

The activation of coagulation detected as thrombin formation (prothrombin fragment $1+2$; F1+2) (Figure 2a) was strongest for glass, followed by PEI whereas values for Teflon AF and SAM-COOH were only about $10 \%$ of the values for PEI. Additionally, TAT (thrombinantithrombin-complex) was determined and showed the same trend as F1+2 (Figure S2). Fibrin formation was determined by detecting fibrinopeptide A (FPA) after cleavage from fibrinogen $\alpha$ chains. Elevated levels of FPA were detected on PEI and glass while SAM$\mathrm{COOH}$ and Teflon AF showed very low values (Figure 2b). This correlates well to the observed macroscopic clot formation (Figure 1).

A decrease in platelet number (Figure 2c) results mainly from adhesion and conjugate/aggregate formation and was highest for glass and significantly increased compared to Teflon AF + SAM-COOH for PEI. As adhesion and activation induce the release of platelet derived mediators like platelet factor 4 (PF4) higher PF4 release was consequently also seen for glass and PEI compared to Teflon AF and $\mathrm{COOH}$ (Figure 2d). 

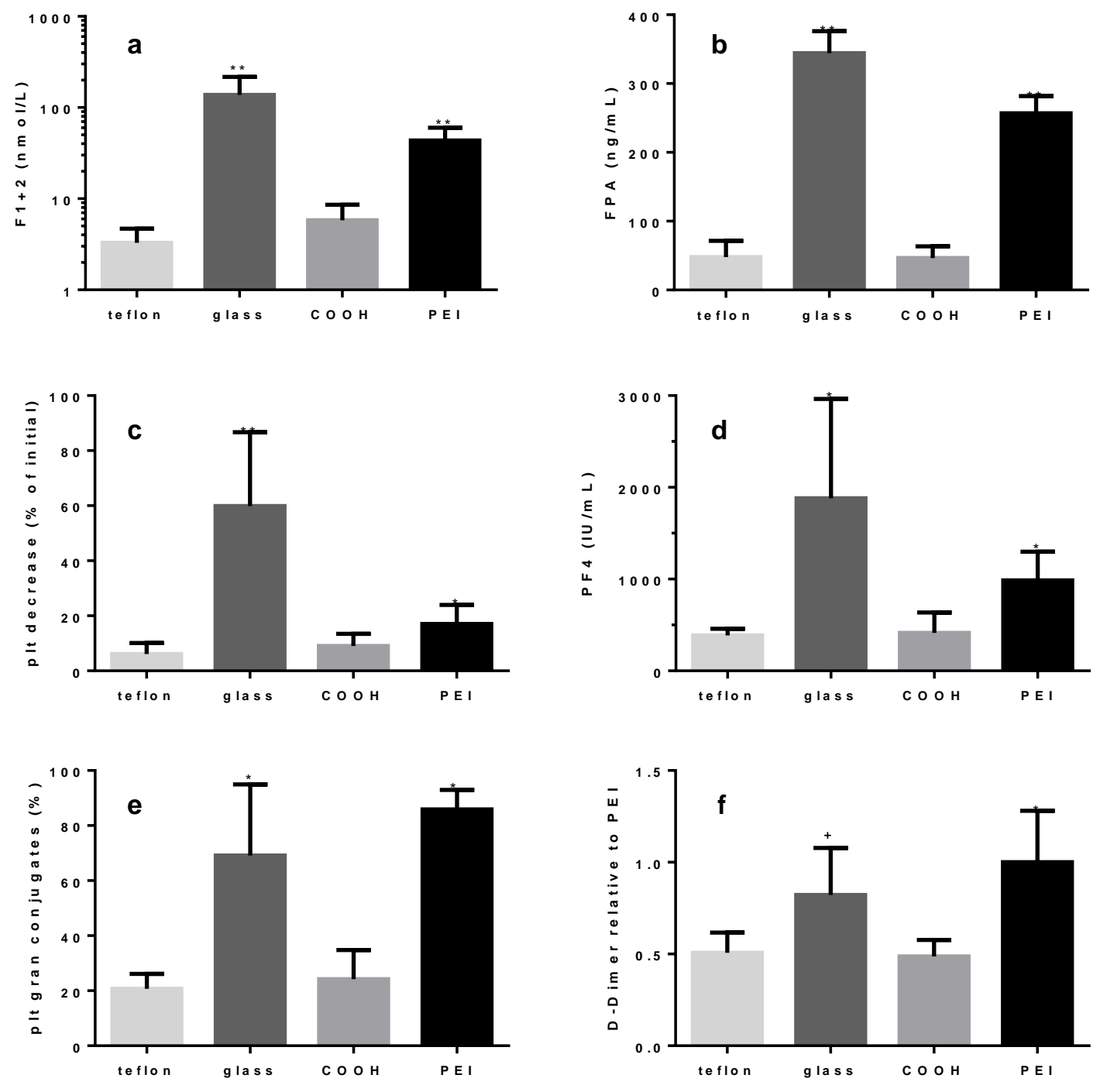

Figure 2. Activation of coagulation, fibrinolysis and platelets. Incubation of surfaces with whole blood (3 hours at $37^{\circ} \mathrm{C}, 1.7 \mathrm{IU}$ heparin). a) Thrombin activation was measured as $\mathrm{F} 1+2$ fragment; b) fibrin formation measured as fibrinopeptide A (FPA); c) decrease in platelet number is given in percentage of the initial value; d) platelet activation measured as PF4 release; e) formation of platelet-granulocyte conjugates; f) D-Dimer formation: activation of fibrinolytic system.

Statistics: *significantly different to Teflon AF and $\mathrm{COOH}, * *$ significantly different to all other samples; +significantly different to $\mathrm{COOH} ; \mathrm{n}=6, \mathrm{n}=3$ for FPA.

The formation of platelet-leukocyte conjugates is known to depend on P-selectin (CD62P) expression on the platelets ${ }^{57}$ and this was highest for PEI and glass (Figure 2e). Conjugate formation was significantly lower for Teflon AF and SAM-COOH. Taken together, these results show that activation of coagulation is highest on glass, strong for PEI surfaces and significantly lower for Teflon AF and SAM-COOH. These results also are reflected on the microscopic pictures of cell adhesion as well as macroscopic clot formation on various surfaces (Figure 1). 
Activation of fibrinolysis was determined by measuring D-Dimer, a degradation product of fibrin, and was found to be elevated on PEI as can be seen in Figure 2f. PEI showed 1.3 times stronger D-Dimer formation compared to glass.

\subsection{Activation of the contact phase of plasmatic coagulation}

Contact activation is a known surface initiated procoagulant reaction through the autoactivation of FXII to FXIIa. The activated enzyme either stays adsorbed to the surface or is released in to the plasma. We, therefore, determined contact activation after incubation of citrated plasma on the surfaces as well as in the liquid phase (Figure 3). Teflon AF and PEI showed no contact activation whereas a strong activation was achieved on the hydrophilic negatively charged surfaces glass and SAM-COOH (Figure 3a). While contact activation measured in plasma was similar for glass and SAM-COOH, the activity on the glass surface itself was significantly higher than on the SAM-COOH surface (Figure 3
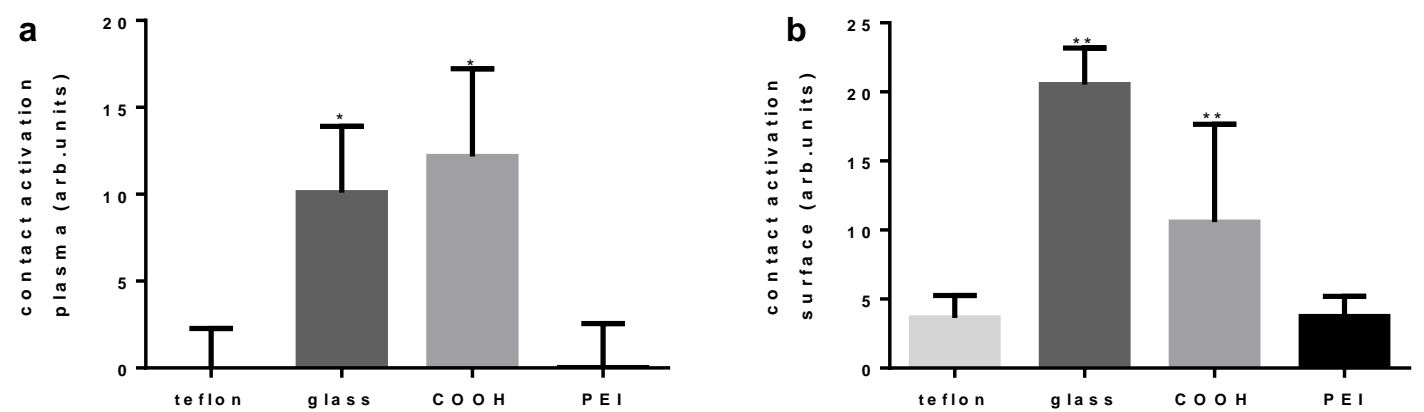

Figure 3. Contact phase activation. Activity of FXIIa and kallikrein measured after plasma incubation with surfaces using the chromogenic substrate S $2302(0.3 \mathrm{mM})$. a) activity in plasma; b) activity on material surfaces. Statistics: ** significantly different to all other surfaces, * significantly different to Teflon AF and PEI.

\subsection{FSAP presence and activity on different surfaces}

To analyse the possible role of FSAP in these pro-coagulant processes, its presence and activation in plasma was determined after incubation with different surfaces (Figure 4a+b). FSAP concentration in plasma showed no material- or time-dependent differences, which excludes major surface-related changes in the adsorption or degradation of FSAP (Figure 4a). For the detection of FSAP activity two independent assays were used: One assay detects the active fraction of FSAP that activates pro-uPA to active $u$-PA (Figure $4 b+d$ ) while the other assay measures the formation of inhibitor complexes between active FSAP and $\alpha 2$ antiplasmin (Figure 4c). Significantly more activated FSAP was detected on PEI compared to all other surfaces for all incubation times beyond 5 minutes in plasma (Figure 4b). Only 
baseline activity was observed for SAM-COOH, glass or Teflon AF surfaces at all time points. To test whether FSAP activation is a general principle on all positively charged surfaces we additionally tested another positively charged surface, PLL. FSAP activation was also observed on PLL but was weaker compared to PEI. (Figure S3). This difference in the extent of activation could be due to differences in chain flexibility or the type of amines present on the two surfaces. While PLL only provides primary amines on the PEI surface $75 \%$ are secondary and tertiary amines are present.

Exactly the same pattern of responses was also observed in whole blood (Figure $4 c+d)$. These results suggest a strong and specific activation of FSAP on the PEI surface in plasma as well as whole blood. FSAP adsorption to the PEI was detected after incubation with whole blood: Western blotting under non-reducing conditions shows FSAP-inhibitor complexes (150-250 $\mathrm{kDa}$ ) as well as intact FSAP (zymogen, $64 \mathrm{kDa}$ ) (Figure 4E). Under reducing conditions, apart from FSAP zymogen, heavy chain $(50 \mathrm{kDa})$ and light chain $(28 \mathrm{kDa})$ were observed (Figure 4F). FSAP zymogen, activated FSAP as well as FSAP inhibitor complexes were adsorbed predominantly on the PEI surface which is congruent with the fact that this surface also activated FSAP strongly. Thus, FSAP has a distinct activation profile compared to the FXII-dependent contact pathway.
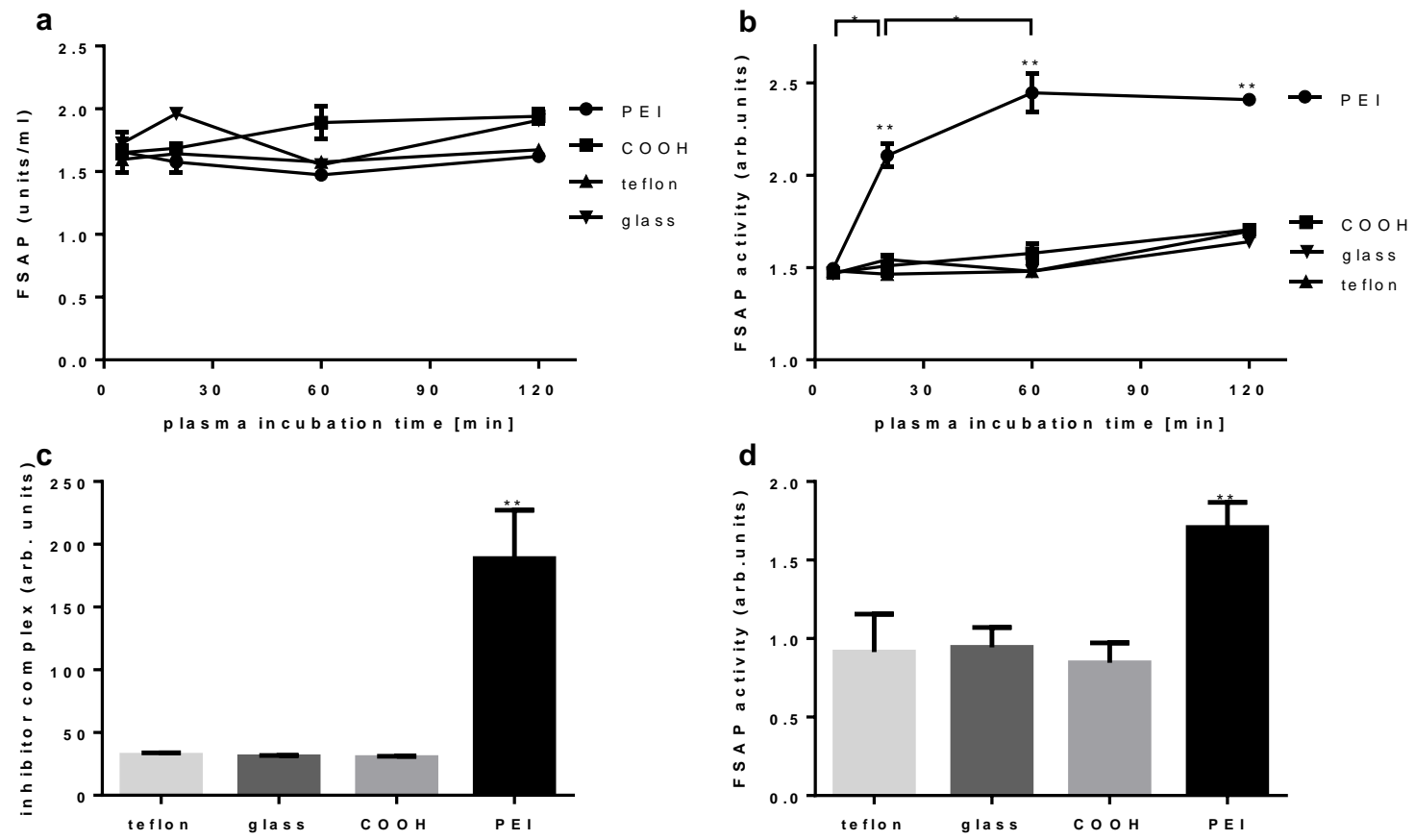


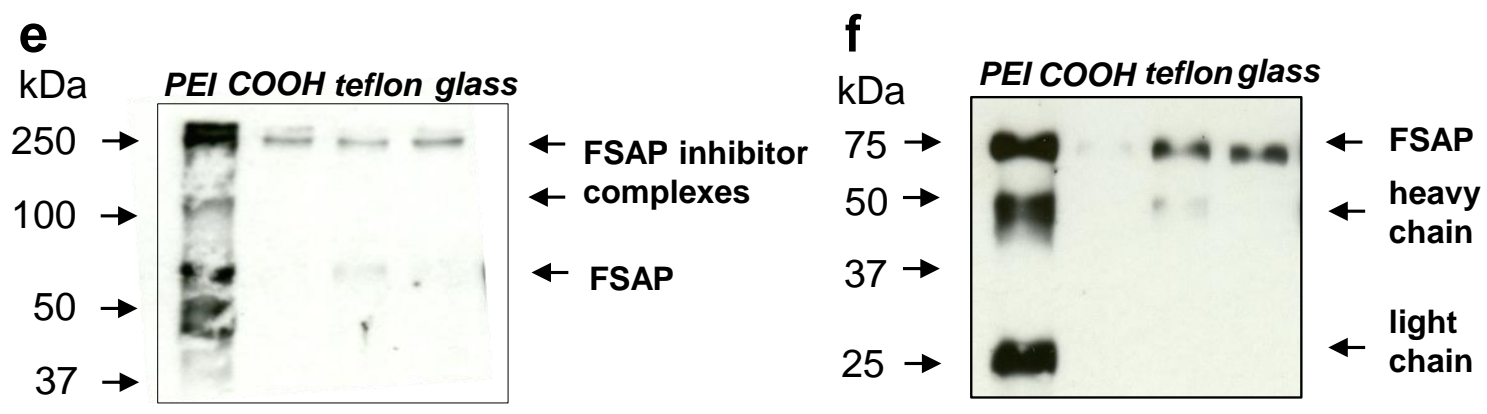

Figure 4. a-d: FSAP presence and activity in blood plasma and blood; e+f: Western blot of FSAP and FSAP inhibitor complex. a-d: FSAP antigen and activity in blood plasma after time dependent incubation (5, 20, 60 or $120 \mathrm{~min}$ ) (a, b) as well as FSAP activity in whole blood after 3 hours of incubation (c,d) on different surfaces: a) FSAP concentration in plasma, b) FSAP activity measured through u-PA activation detection using chromogenic assay (mean $\pm \mathrm{SD}, \mathrm{n}=2$ ); c) detection of FSAP- $\alpha 2$-antiplasmin-complex; d) detection of $\mathrm{u}$-PA activation through activated FSAP; Statistics: (b)** significantly different to all other samples at this time-point, *significantly different to other time points of PEI, $(\mathrm{c}+\mathrm{d}) * *$ significantly different to all other samples.

$(\mathrm{e}+\mathrm{f})$ : Western blot of FSAP and FSAP inhibitor complex after in vitro whole blood incubation of surfaces.

Detection using mouse monoclonal antibodies for FSAP heavy and light chain; (e) non-reducing conditions; (f) reducing conditions.

3.4. Blood incubation using inhibitory monoclonal antibody (mab)570 or contact phase inhibitor CTI

To further define the role of FSAP and FXIIa on surface-mediated coagulation, an inhibitory antibody to FSAP, mab570, and an inhibitor to FXIIa was used. Figure 5a shows that mab570 did not influence thrombin formation on Teflon AF, glass and SAM-COOH, but there was a clear decrease on the PEI surface with mab570 compared to the isotype control antibody IgG. The FXIIa inhibitor CTI on the other hand reduced thrombin formation distinctively on glass and to a lower extent also on Teflon AF and SAM-COOH but not on PEI (Figure 5b). This result suggests that on the cationic surface PEI FSAP but not contact activation is relevant for thrombin formation.
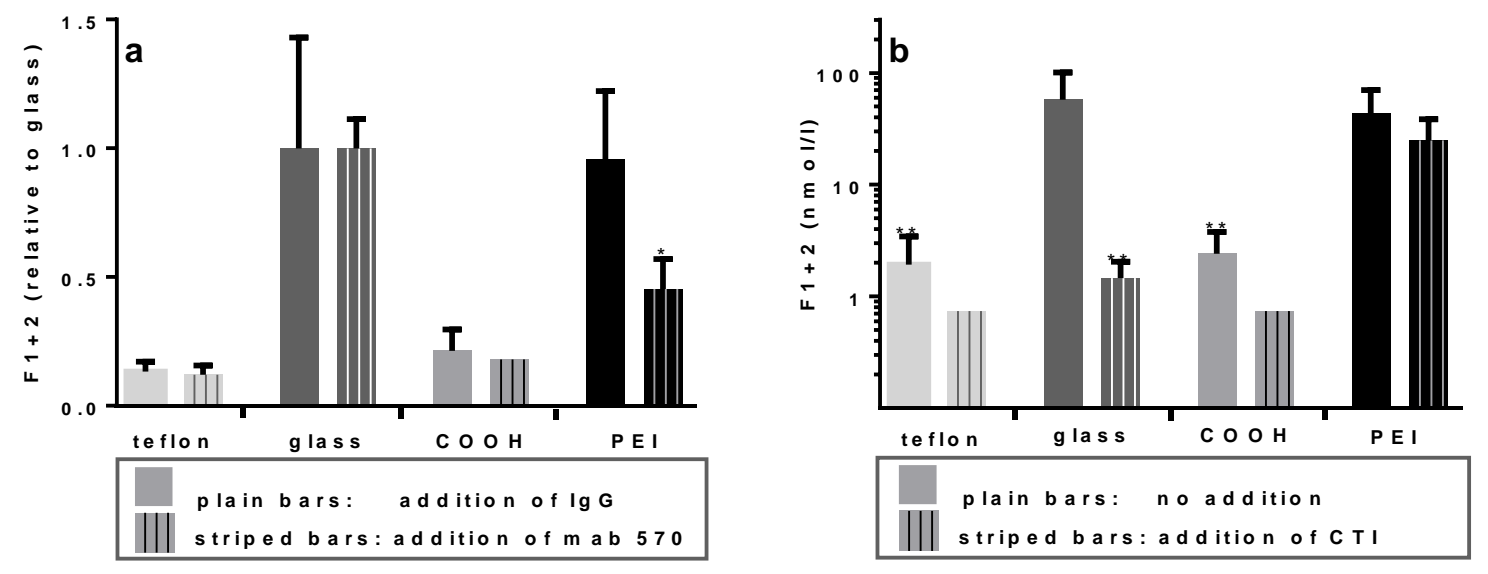
Figure 5. Coagulation activation on surfaces with inhibitors to FSAP (a) or the contact phase (b). a: addition of inhibitory FSAP antibody mab570 to the blood (c=100 $\mu \mathrm{g} / \mathrm{mL})$ before incubation to block endogenous FSAP compared to control IgG isotype antibody $(100 \mu \mathrm{g} / \mathrm{mL})$. Statistics: * significantly different to PEI IgG (unpaired, one tailed t-test). b: addition of contact phase inhibitor CTI ( $\mathrm{c}=10 \mathrm{U} / \mathrm{mL}$ ) before incubation compared to addition of PBS (same volume). Statistics: ** significantly different to glass w/o CTI and PEI w/o CTI.

\subsection{Heparin interaction with surfaces}

Neutralization of the polyanion heparin by the positively charged surfaces may account for the pro-coagulant effects of the PEI surface. We determined heparin concentration in blood / plasma (citrated, heparin supplementation) after incubation with surfaces. At the given geometries, the PEI surface adsorbed heparin and lowered the free heparin concentration from $1.7 \mathrm{IU} / \mathrm{mL}$ to $1.2 \mathrm{IU} / \mathrm{mL}$ (Figure $\mathrm{S} 4$ ), confirming previous observations. ${ }^{25}$ Based on these results a subsequent whole blood incubation with Teflon AF samples was done with the correspondingly adjusted heparinization of $1.2 \mathrm{IU} / \mathrm{mL}$ heparin compared to the original heparinization of $1.7 \mathrm{IU} / \mathrm{mL}$. Apart from the change in heparin concentration these experiments were performed as described in the relevant methods section. This reduction in the heparin content did induce minor differences for thrombin formation, PF4 formation and complement. Thrombin formation was slightly elevated in the samples with the lower heparin content: $4.0 \pm 0.6$ versus $1.8 \pm 0.8 \mathrm{nmol} / \mathrm{L}$, both values close to the previously measured $3.3 \pm 1.4$ $\mathrm{nmol} / \mathrm{L}$ for Teflon AF but much lower compared to $50 \pm 0.7 \mathrm{nmol} / \mathrm{L}$ that were measured for PEI. These results illustrate that a decrease in heparin content on PEI only induces minor changes in coagulation parameters as long as no additional activation pathway is initiated. Thus, the minor sequestering of heparin on PEI cannot account for the strong FSAP activation and procoagulant response on this surface.

\section{Activation of immunologic reactions}

As complement activation can be influenced by cellular processes in blood and in turn can influence coagulation, we determined complement activation in serum as well as whole blood after incubation with different surfaces. In serum, significantly enhanced values for C5a formation as well as for $\mathrm{C} 3 \mathrm{~b}$ and $\mathrm{C} 4 \mathrm{~d}$ adsorption to PEI surfaces was observed (Figure S5). The other surfaces did not show differences for $\mathrm{C} 5 \mathrm{a}$ and $\mathrm{C} 4 \mathrm{~d}$ while for $\mathrm{C} 3 \mathrm{~b}$ minor differences were seen with Teflon AF inducing the lowest and SAM-COOH the highest values.

After whole blood incubation C5a formation was enhanced on glass and to a lesser extent on SAM-COOH while PEI and Teflon AF showed minimal activation (Figure S5d). C3b 
adsorption was strongest for PEI (Figure S5e), intermediate on glass and $\mathrm{COOH}$ while significantly less C3b was found on Teflon AF.

High levels of granulocyte activation were observed on all surfaces, except Teflon AF (Figure S5f). As the high activation at the SAM-COOH and PEI surface cannot be explained by the C5a anaphylatoxin, additional activators seem to be relevant.

\section{Discussion}

The current paradigm for the activation of coagulation by biomaterials is based on the concept of FXII auto-activation on negatively charged surfaces and the subsequent activation of the intrinsic pathway. ${ }^{58}$ However, it has also been known for a long time that positively charged surfaces activate coagulation to a similar extent as negatively charged surfaces. While the molecular mechanisms of the contact phase of coagulation activation at anionic surfaces are quite well understood ${ }^{11,59}$ the same is not the case for positively charged surfaces.

To redress this, we examined a set of model surfaces including positively charged polyethylenimine (PEI) and poly-L-lysine (PLL) layers, negatively charged glass, selfassembled organic monolayers exposing carboxylic acid groups (SAM-COOH) as well as a hydrophobic fluoropolymer (Teflon AF) with respect to the activation of coagulation, complement and immune reactions.

As expected, the contact phase of the coagulation cascade was only activated on negatively charged surfaces (SAM-COOH, glass), but not on Teflon-AF or PEI. On SAM-COOH the strong contact phase activation cannot be propagated sufficiently to a coagulation cascade due to the lack of other pro-coagulant supporters like adherent platelets, as shown in a previous study. ${ }^{11}$ Thus, coagulation on this surface remained low. On glass, the strong contact activation combined with stimulation of humoral and cellular inflammation caused the highest coagulation activation during the whole blood incubation, as measured by thrombin $(\mathrm{F} 1+2)$ and platelet activation (PF4). This is further supported by the fact that the contact pathway inhibitior, CTI, diminished coagulation activation on glass to levels comparable to Teflon AF.

On Teflon AF no contact activation was seen. While some authors see contact activation related to hydrophilicity and negative surface charge, ${ }^{60}$ Vogler and his group claim that this 
effect is only due to an "apparent specificity (of FXII) for hydrophilic surfaces that is actually due to a relative diminution of activation at hydrophobic surfaces". ${ }^{13}$ This conclusion was drawn from work with isolated proteins. Yet, in complex media like plasma and blood competing protein adsorption processes interfere with contact activation and the resulting plasmatic coagulation is low on hydrophobic surfaces. Teflon AF on the other hand strongly supports platelet adhesion as can be seen with SEM, yet granular release (PF4 release) is low. Normally platelet adhesion and platelet aggregation can be seen as two sides of the same coin. ${ }^{61}$ In the blood vessel platelet adhesion is realized on the extracellular matrix consisting of different types of adhesive proteins and several different adhesion receptors are involved. In the case of biomaterials this complexity is missing: mainly adsorbed, conformational changed fibrinogen is responsible for the adhesion of platelets with $\mathrm{vWF}^{62}$ possibly being important for in vitro incubation under (moderate to high) shear stress. ${ }^{61}$

The positively charged PEI surface induced thrombin activation as well as platelet activation during the whole blood incubation almost to the same level as the negatively charged glass. Surface-mediated activation of FSAP was found only on positively charged PEI and PLL. PLL also supported the activation of FSAP activation but the effect was weaker. This indicates that not just charge per se, but also density and type of functional group and flexibility of the molecules determines the specificity of this reaction. An effect of surface hydrophobicity - as discussed above for FXIIa - can be ruled out, as neither the hydrophilic glass and SAM-COOH surface nor the hydrophobic Teflon AF induced FSAP activation. The activation of FSAP was distinct and reproducible in plasma as well as in human whole blood (blood incubation was only performed for PEI); therefore a direct correlation with coagulation activation seems probable. Although neutralization of the polyanion heparin on this surface was present, this had only a minor contribution to the elevated coagulation at the PEI surface. Control experiments with an inhibitory antibody against FSAP confirmed a major contribution of FSAP to the thrombin activation by this positively charged surface. Additionally CTI was applied to study contact phase activation. While almost completely abrogating F1+2 formation on glass only marginal effects were seen on PEI. This implies that contact activation is not accountable for the activation of coagulation on PEI.

These results possibly demonstrate a new mechanism for coagulation activation triggered by positively charged surfaces. For the first time, the activation of the plasmatic zymogen FSAP by a macroscopic polymeric material with the subsequent activation of coagulation was demonstrated. The mechanism appears to be the cationic counterpart to the well-described 
contact-phase activation at anionic surfaces. Inhibition of FSAP decreased thrombin activation and fibrin generation on PEI but it was not clear exactly how the extrinsic pathway was activated in the absence of contact activation. Although whole blood incubation would have sufficient TF, provided by blood cells and platelet and monocyte derived microparticles, ${ }^{63-67}$ in plasma only microparticles are present. In case of co-incubation of plasma with endothelial cells FSAP could inhibit TFPI and thus increase FVIIa activity and TF-dependent FXa generation. ${ }^{34}$ TF also is released from leukocytes upon activation at foreign surfaces. ${ }^{65,68}$ FSAP mediated TFPI inhibition also here can enhance the impact of this pathway on FXa generation. In the case of material surfaces we have not detected changes in TFPI activity in the solution phase on various surfaces (unpublished data) but the possibility that FSAP has effects on other coagulation components remains. Thus, we speculate that the observed effects on PEI are related to the activation of FSAP that trigger coagulation independently of known pathways.

The pro-coagulant effect of the surface-immobilized PEI appears to be in contrast to former studies, which showed an anticoagulant effect of soluble PEI. These experiments were performed using isolated proteins ${ }^{69,70}$ and soluble PEI which directly interacts with coagulation factors and prevents cleavage of fibrinogen. These mechanisms cannot occur for the surface-immobilized molecule in the present study. Additionally the interaction of FSAP with nanomaterial surfaces might be different depending on nanomaterials size and the resulting curvature as this was reported to be important protein adsorption on the whole. ${ }^{71}$ Since the activation process of FSAP on charged surfaces certainly depends on the specific interaction with the surface only additional experiments with charged nanoparticles could clarify the influence of curvature on FSAP surface interactions. A hindrance of coagulation complexes formation on nanomaterials due to lack of space on small nanoparticles has been reported and could be an additional confounding factor. ${ }^{72}$

Since FSAP has been reported to activate multiple pathways, the impact of the surface activated FSAP on other processes besides coagulation was also determined. Activation of fibrinolysis by activation of u-PA had been described. ${ }^{30}$ While in the present study the formation of fibrin (determined through FPA detection) was strongest for glass surfaces, corresponding to the high thrombin activation, the strongest D-Dimer formation was seen for PEI. Positively charged, lysine exposing surfaces have been shown to promote fibrinolysis by binding plasminogen and tissue plasminogen activator (t-PA). ${ }^{73,74}$ This nevertheless was not 
described to be generally applicable to other positively charged surfaces. Therefore the slightly enhanced fibrinolysis could possibly be attributed to FSAP.

The activation of the complement system by materials surfaces is often attributed to nucleophilic groups $\left(-\mathrm{OH},-\mathrm{NH}_{2}\right)$ that support $\mathrm{C} 3$ hydrolysis. Complement activation through FSAP has also been described in vitro. ${ }^{41}$ In a previous study in plasma of trauma patients a correlation between FSAP activation and C5a levels but not C3a levels was observed. Additionally the activation of FSAP in the plasma led to the generation of C5a. ${ }^{41}$ In our study however the results of complement and immunologic activation were ambiguous. The detection of complement activation in serum showed a significantly higher activation of complement on PEI surfaces: $\mathrm{C} 3 \mathrm{~b}$ and $\mathrm{C} 4 \mathrm{~d}$ adsorption was highest on PEI as was $\mathrm{C} 5 \mathrm{a}$ generation. Yet complement activation in blood was only elevated on glass, not on the FSAP activating surface. Glass is a hydrophilic material which offers hydroxyl groups on the surface leading to complement activation through a covalent bonding of $\mathrm{C} 3 \mathrm{~b}$. On negatively charged SAM-COOH surface $\mathrm{C} 3 \mathrm{~b}$ activity probably is regulated by inhibitory complement factor $\mathrm{H}$ which competes with factor $\mathrm{B}$ in binding to $\mathrm{C} 3 \mathrm{~b} .{ }^{75}$ Comparably, negatively charged heparin adsorbed to PEI surfaces (Figure S3) could induce complement inhibitory reactions via the adsorption of factor $\mathrm{H}$. A direct activation of complement through amine groups on the PEI surface is possible as well, however, $-\mathrm{NH}_{2}$ groups were reported to support the activation of complement with a low efficiency only. ${ }^{16}$ On the whole complement activation on FSAP activating materials was not robust and deserves further experimental clarification.

Previous studies indicate that both positive and negative charged macromolecules like nucleic acids and histones activate FSAP zymogen. ${ }^{38,39,76}$ For either type of ionized polymer, a template effect is proposed whereas soluble positive or negative charges help to unblock the E3 domain (see Figure 6 center) which allows autoactivation. For reasons that are not completely clear only positively charged macromolecules activate FSAP zymogen in plasma. ${ }^{41}$ Here we show that also extended flat negatively charged surfaces do not activate FSAP in plasma which is compatible with these previous observations. Probably, the proposed conformational change on negatively charged molecules and therefore also surfaces requires a specific molecular conformation or charge density. Additionally other plasma proteins may interfere with the FSAP activation process in the case of negatively charged protein. On the other hand we found that surfaces with positively charged surfaces effectively can induce autoactivation comparably to positively charged macromolecules (Figure 6). 


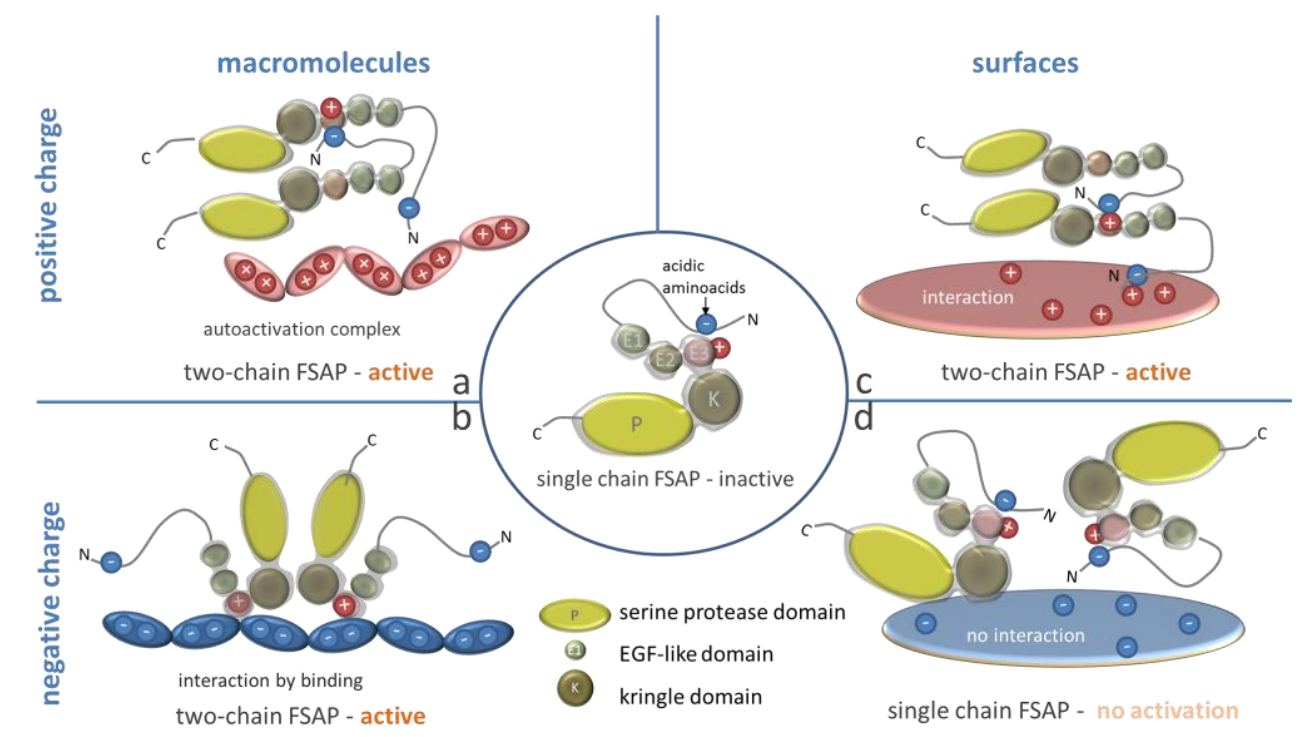

Figure 6 Proposed process of FSAP interaction with positive and negatively charged macromolecules / surfaces. Center: single FSAP molecule, proposed interaction of FSAP with charged macromolecules (left side, $\mathrm{a}+\mathrm{b}$ ) and extended planar material surfaces (right side, $\mathrm{c}+\mathrm{d}$ ) with positive charge (top row, $\mathrm{a}+\mathrm{c}$ ) or negative charge (bottom row, $b+d)$. (Adapted from Yamamichi et al. ${ }^{39}$ )

\section{CONCLUSION}

Activation of plasma- or blood-derived FSAP is induced on positively charged surfaces such as PEI and this is associated with a strong pro-coagulant response. A significant reduction of coagulation was achieved on PEI with the inhibition of FSAP while the inhibition of FXII had no effect. We propose a novel mechanism of surface-driven coagulation important for understanding the hemo(in)compatibity of PEI, a positively charged biomedical material. The reported findings clarify an important aspect of the interaction of blood with medical devices and implants and further expand our understanding of the role of FSAP in coagulation and immunology. A therapeutic concept to limit FSAP induced blood activation with inhibitors might be feasible and possibly would expand the potential application of cationic materials for medical use.

SUPPORTING INFORMATION: Quantification of platelet adhesion to surfaces; Comparison of thrombin formation parameter F1+2 and TAT, Determination of FSAP- $\alpha 2-$ antiplasmin-complex in blood plasma after incubation on different surfaces; Heparin content in blood and in plasma after in vitro incubation of surfaces; Activation of immunologic reactions after incubation of surfaces with serum or whole blood 


\section{AUTHOR INFORMATION}

Corresponding author

*Corresponding author: Claudia Sperling, Ph.D., Institute Biofunctional Polymer Materials, Max Bergmann Center of Biomaterials, Leibniz-Institut für Polymerforschung Dresden e.V., Hohe Str. 6, 01069 Dresden, Germany

Tel.: +49-351-4658-274

Fax: +49-351-4658-533

E-mail: sperling@ipfdd.de

Conflict of interest

The authors declare no conflict of interest.

Acknowledgements

The authors thank Monique Marx, Stefanie Hänsel, Martina Franke, Lydia Neubeck and Aurelia Laubscher for practical assistance in experimental work concerning surface preparation, blood incubation, plasma and surface analysis. We also thank Marion Fischer for help with data interpretation. Additionally we want to thank Michael Etscheid for critical comments to experimental design and data interpretation.

Authorship contributions

C.S. designed experiments, analyzed data and wrote the manuscript; M.F.M. participated in experimental design, data interpretation and critically revised the manuscript; S.G. and S.M.K. designed and performed experiments for FSAP (activation) detection and Western Blot; S.M.K. additionally participated in general experimental design, data interpretation and manuscript writing; C.W. critically revised the experimental set-up as well as the manuscript. All authors have given approval to the final version of the manuscript.

\section{ABBREVIATIONS}

FSAP: Factor VII activating protease; PEI: polyethylenimine; PLL: poly-L-lysine, HABP2: hyaluronic acid binding protein 2; u-PA: urokinase-type plasminogen activator ; TFPI: tissue factor pathway inhibitor, SAM: self-assembled monolayers; CTI: corn trypsin inhibitor; F1+2: prothrombin fragment 1+2, TAT: thrombin-antithrombin-complex; PF4: platelet factor 4, SEM: scanning electron microscopy; PRP: platelet rich plasma, LDH: lactate dehydrogenase 


\section{REFERENCES}

(1) Gorbet, M. B.; Sefton, M. V. Biomaterial-associated thrombosis: roles of coagulation factors, complement, platelets and leukocytes. Biomaterials 2004, 25, 5681-5703.

(2) Linnemann, B.; Lindhoff-Last, E. Risk factors, management and primary prevention of thrombotic complications related to the use of central venous catheters. Vasa 2012, 41, 319-332.

(3) Lakbakbi, S.; Debrumetz, A.; Terryn, C.; Szymezak, J.; Rieu, P.; Nguyen, P. Tissue factor expressed by adherent cells contributes to hemodialysis-membrane thrombogenicity. Thromb. Res. 2016, 144, 218-223.

(4) Liu, X. L.; Yuan, L.; Li, D.; Tang, Z. C.; Wang, Y. W.; Chen, G. J.; Chen, H.; Brash, J. L. Blood compatible materials: state of the art. J. Mat. Chem. B 2014, 2, 5718-5738.

(5) Werner, C.; Maitz, M. F.; Sperling, C. Current strategies towards hemocompatible coatings. J. Mater. Chem. 2007, 17, 3376-3384.

(6) Barnes, L.; Cooper, I., Biomaterials and Medical Device - Associated Infections. Elsevier Science: 2014.

(7) Vroman, L. The life of an artificial device in contact with blood. Bull.N.Y.Acad.Med. 1988, 64, 352357.

(8) Rodrigues, S. N.; Goncalves, I. C.; Martins, M. C.; Barbosa, M. A.; Ratner, B. D. Fibrinogen adsorption, platelet adhesion and activation on mixed hydroxyl-/methyl-terminated self-assembled monolayers. Biomaterials 2006, 27, 5357-5367.

(9) Hylton, D. M.; Shalaby, S. W.; Latour, R. A., Jr. Direct correlation between adsorption-induced changes in protein structure and platelet adhesion. J. Biomed. Mater. Res. A 2005, 73, 349-358.

(10) Sivaraman, B.; Latour, R. A. The relationship between platelet adhesion on surfaces and the structure versus the amount of adsorbed fibrinogen. Biomaterials 2010, 31, 832-839.

(11) Sperling, C.; Fischer, M.; Maitz, M. F.; Werner, C. Blood coagulation on biomaterials requires the combination of distinct activation processes. Biomaterials 2009, 30, 4447-4456.

(12) Yau, J. W.; Liao, P.; Fredenburgh, J. C.; Stafford, A. R.; Revenko, A. S.; Monia, B. P.; Weitz, J. I. Selective depletion of factor XI or factor XII with antisense oligonucleotides attenuates catheter thrombosis in rabbits. Blood 2014, 123, 2102-2107.

(13) Zhuo, R.; Siedlecki, C. A.; Vogler, E. A. Competitive-protein adsorption in contact activation of blood factor XII. Biomaterials 2007, 28, 4355-4369.

(14) Zhuo, R.; Siedlecki, C. A.; Vogler, E. A. Autoactivation of blood factor XII at hydrophilic and hydrophobic surfaces. Biomaterials 2006, 27, 4325-4332.

(15) Vroman, L. Finding seconds count after contact with blood (and that is all I did). Coll Surf $B$ : Biointerfaces 2008, 62, 1-4.

(16) Toda, M.; Kitazawa, T.; Hirata, I.; Hirano, Y.; Iwata, H. Complement activation on surfaces carrying amino groups. Biomaterials 2008, 29, 407-417.

(17) Sperling, C.; Maitz, M. F.; Talkenberger, S.; Gouzy, M. F.; Groth, T.; Werner, C. In vitro blood reactivity to hydroxylated and non-hydroxylated polymer surfaces. Biomaterials 2007, 28, 36173625.

(18) Engberg, A. E.; Rosengren-Holmberg, J. P.; Chen, H.; Nilsson, B.; Lambris, J. D.; Nicholls, I. A.; Ekdahl, K. N. Blood protein-polymer adsorption: Implications for understanding complementmediated hemoincompatibility. J. Biomed. Mater. Res. A 2011, 97A, 74-84.

(19) Andersson, J.; Ekdahl, K. N.; Lambris, J. D.; Nilsson, B. Binding of C3 fragments on top of adsorbed plasma proteins during complement activation on a model biomaterial surface.

Biomaterials 2005, 26, 1477-1485.

(20) Nilsson, B.; Ekdahl, K. N.; Mollnes, T. E.; Lambris, J. D. The role of complement in biomaterialinduced inflammation. Mol. Immunol. 2007, 44, 82-94.

(21) Ghasemzadeh, M.; Hosseini, E. Platelet-leukocyte crosstalk: Linking proinflammatory responses to procoagulant state. Thromb. Res. 2013, 131, 191-197. 
(22) Miyamoto, M.; Sasakawa, S.; Ozawa, T.; Kawaguchi, H.; Ohtsuka, Y. Mechanisms of blood coagulation induced by latex particles and the roles of blood cells. Biomaterials 1990, 11, 385-388.

(23) Sagnella, S.; Mai-Ngam, K. Chitosan based surfactant polymers designed to improve blood compatibility on biomaterials. Coll. Surf. B: Biointerfaces 2005, 42, 147-155.

(24) Jones, C. F.; Campbell, R. A.; Franks, Z.; Gibson, C. C.; Thiagarajan, G.; Vieira-de-Abreu, A.; Sukavaneshvar, S.; Mohammad, S. F.; Li, D. Y.; Ghandehari, H.; Weyrich, A. S.; Brooks, B. D.; Grainger, D. W. Cationic PAMAM dendrimers disrupt key platelet functions. Mol Pharm 2012, 9, 1599-1611.

(25) Schulze, A.; Maitz, M. F.; Zimmermann, R.; Marquardt, B.; Fischer, M.; Werner, C.; Went, M.; Thomas, I. Permanent surface modification by electron-beam-induced grafting of hydrophilic polymers to PVDF membranes. RSC Advances 2013, 3, 22518-22526.

(26) Jones, C. F.; Campbell, R. A.; Brooks, A. E.; Assemi, S.; Tadjiki, S.; Thiagarajan, G.; Mulcock, C.; Weyrich, A. S.; Brooks, B. D.; Ghandehari, H.; Grainger, D. W. Cationic PAMAM dendrimers aggressively initiate blood clot formation. ACS Nano 2012, 6, 9900-9910.

(27) Bilensoy, E. Cationic nanoparticles for cancer therapy. Expert Opin. Drug Deliv. 2010, 7, 795809.

(28) Römisch, J.; Feussner, A.; Vermöhlen, S.; Stöhr, H. A. A protease isolated from human plasma activating factor VII independent of tissue factor. Blood. Coagul. Fibrinolysis 1999, 10, 471-479.

(29) Römisch, J. Factor VII activating protease (FSAP): a novel protease in hemostasis. Biol.Chem. 2002, 383, 1119-1124.

(30) Römisch, J.; Feussner, A.; Vermöhlen, S.; Stöhr, H. A. The FVII Activating Protease Cleaves Single-Chain Plasminogen Activators. Pathophysiol Haemos Thromb 1999, 29, $292-299$.

(31) Stavenuiter, F.; Dienava-Verdoold, I.; Boon-Spijker, M. G.; Brinkman, H. J. M.; Meijer, A. B.; Mertens, K. Factor seven activating protease (FSAP): does it activate factor VII? J. Thromb. Haemost. 2012, 10, 859-866.

(32) Khandekar, G.; Jagadeeswaran, P. Role of hepsin in factor VII activation in zebrafish. Blood Cells, Mol. Dis. 2014, 52, 76-81.

(33) Subramaniam, S.; Thielmann, I.; Morowski, M.; Pragst, I.; Sandset, P. M.; Nieswandt, B.; Etscheid, M.; Kanse, S. M. Defective thrombus formation in mice lacking endogenous factor VII activating protease (FSAP). Thromb. Haemost. 2015, 113, 870-880.

(34) Kanse, S. M.; Declerck, P. J.; Ruf, W.; Broze, G.; Etscheid, M. Factor VII-activating protease promotes the proteolysis and inhibition of tissue factor pathway inhibitor. Arterioscler. Thromb. Vasc. Biol. 2012, 32, 427-433.

(35) Wood, J. P.; Ellery, P. E.; Maroney, S. A.; Mast, A. E. Biology of tissue factor pathway inhibitor. Blood 2014, 123, 2934-2943.

(36) Wood, J. P.; Bunce, M. W.; Maroney, S. A.; Tracy, P. B.; Camire, R. M.; Mast, A. E. Tissue factor pathway inhibitor-alpha inhibits prothrombinase during the initiation of blood coagulation. Proc. Natl. Acad. Sci. U. S. A. 2013, 110, 17838-17843.

(37) Kannemeier, C.; Feussner, A.; Stöhr, H. A.; Weisse, J.; Preissner, K. T.; Römisch, J. Factor VII and single-chain plasminogen activator-activating protease: activation and autoactivation of the proenzyme. Eur. J. Biochem. 2001, 268, 3789-3796.

(38) Altincicek, B.; Shibamiya, A.; Trusheim, H.; Tzima, E.; Niepmann, M.; Linder, D.; Preissner, K. T.; Kanse, S. M. A positively charged cluster in the epidermal growth factor-like domain of Factor VIIactivating protease (FSAP) is essential for polyanion binding. Biochem. J. 2006, 394, 687-692.

(39) Yamamichi, S.; Nishitani, M.; Nishimura, N.; Matsushita, Y.; Hasumi, K. Polyamine-promoted autoactivation of plasma hyaluronan-binding protein. J. Thromb. Haemost. 2010, 8, 559-566.

(40) Yamamichi, S.; Fujiwara, Y.; Kikuchi, T.; Nishitani, M.; Matsushita, Y.; Hasumi, K. Extracellular histone induces plasma hyaluronan-binding protein (factor VII activating protease) activation in vivo. Biochem. Biophys. Res. Commun. 2011, 409, 483-488.

(41) Kanse, S. M.; Gallenmueller, A.; Zeerleder, S.; Stephan, F.; Rannou, O.; Denk, S.; Etscheid, M.; Lochnit, G.; Krueger, M.; Huber-Lang, M. Factor VII-Activating Protease is activated in multiple trauma patients and generates anaphylatoxin C5a. J/ 2012, 188, 2858-2865. 
(42) Choi-Miura, N. H.; Takahashi, K.; Yoda, M.; Saito, K.; Mazda, T.; Tomita, M. Proteolytic activation and inactivation of the serine protease activity of plasma hyaluronan binding protein. Biol. Pharm. Bull. 2001, 24, 448-452.

(43) Parahuleva, M. S.; Maj, R.; Hälschermann, H.; Parviz, B.; Abdallah, Y.; Erdogan, A.; Tillmanns, H.; Kanse, S. M. Regulation of monocyte/macrophage function by factor VII activating protease (FSAP). Atherosclerosis 2013, 230, 365-372.

(44) Stephan, F.; Hazelzet, J. A.; Bulder, I.; Boermeester, M. A.; van Till, J. O.; van der Poll, T.; Wuillemin, W. A.; Aarden, L. A.; Zeerleder, S. Activation of factor VII-activating protease in human inflammation: a sensor for cell death. Crit. Care 2011, 15, R110.

(45) Yamano, S.; Dai, J.; Hanatani, S.; Haku, K.; Yamanaka, T.; Ishioka, M.; Takayama, T.; Yuvienco, C.; Khapli, S.; Moursi, A. M.; Montclare, J. K. Long-term efficient gene delivery using polyethylenimine with modified Tat peptide. Biomaterials 2014, 35, 1705-1715.

(46) Fischer, M.; Sperling, C.; Werner, C. Synergistic effect of hydrophobic and anionic surface groups triggers blood coagulation in vitro. J. Mater. Sci. Mater. Med. 2009, 21, 931-937.

(47) Werner, C.; Zimmermann, R.; Kratzmüller, T. Streaming potential and streaming current measurements at planar solid/liquid interfaces for simultaneous determination of zeta potential and surface conductivity. Colloids Surf. A: Physicochem. Eng. Asp. 2001, 192, 205-213.

(48) Duval, J. F.; Küttner, D.; Nitschke, M.; Werner, C.; Zimmermann, R. Interrelations between charging, structure and electrokinetics of nanometric polyelectrolyte films. J. Colloid. Interface Sci. 2011, 362, 439-449.

(49) Nitschke, M.; Zschoche, S.; Baier, A.; Simon, F.; Werner, C. Low pressure plasma immobilization of thin hydrogel films on polymer surfaces. SurfCoat 2004, 185, 120-125.

(50) Zimmermann, R.; Rein, N.; Werner, C. Water ion adsorption dominates charging at nonpolar polymer surfaces in multivalent electrolytes. Physical Chemistry Chemical Physics 2009, 11, 4360 4364.

(51) Zimmermann, R.; Freudenberg, U.; Schweiss, R.; Kuttner, D.; Werner, C. Hydroxide and hydronium ion adsorption - A survey. Curr. Opin. Colloid. Interface S. 2010, 15, 196-202.

(52) Sabbatovskii, K. G.; Dutschk, V.; Nitschke, M.; Simon, F.; Grundke, K. Properties of the Teflon AF1601S surface treated with the low-pressure argon plasma. Colloid Journal 2004, 66, 208-215.

(53) Nitschke, M.; Gramm, S.; Götze, T.; Valtink, M.; Drichel, J.; Voit, B.; Engelmann, K.; Werner, C. Thermo-responsive poly(NiPAAm-Co-DEGMA) substrates for gentle harvest of human corneal endothelial cell sheets. J. Biomed. Mater. Res. A 2007, 80A, 1003-1010.

(54) Streller, U.; Sperling, C.; Hubner, J.; Hanke, R.; Werner, C. Design and evaluation of novel blood incubation systems for in vitro hemocompatibility assessment of planar solid surfaces. J. Biomed. Mater. Res. 2003, 66B, 379-390.

(55) Yeo, E. L.; Sheppard, J. A.; Feuerstein, I. A. Role of P-selectin and leukocyte activation in polymorphonuclear cell adhesion to surface adherent activated platelets under physiologic shear conditions (an injury vessel wall model). Blood 1994, 83, 2498-2507.

(56) Romisch, J.; Feussner, A.; Stohr, H. A. Quantitation of the factor VII- and single-chain plasminogen activator-activating protease in plasmas of healthy subjects. Blood Coagul Fibrinolysis 2001, 12, 375-383.

(57) Pidard, D. S.-T., M; Chignard, M, Neutrophil-platelet interactions. Harwood Academic Publishers: Amsterdam: 2000; Vol. Platelets, thrombosis and the vessel wall, p p. 189-207.

(58) Zhu, S.; Diamond, S. L. Contact activation of blood coagulation on a defined kaolin/collagen surface in a microfluidic assay. Thromb. Res. 2014, 134, 1335-1343.

(59) Vogler, E. A.; Siedlecki, C. A. Contact activation of blood-plasma coagulation. Biomaterials 2009, 30, 1857-1869.

(60) Sanchez, J.; Lundquist, P. B.; Elgue, G.; Larsson, R.; Olsson, P. Measuring the degree of plasma contact activation induced by artificial materials. Thromb. Res. 2002, 105, 407-412.

(61) Ruggeri, Z. M.; Mendolicchio, G. L. Adhesion Mechanisms in Platelet Function. Circ. Res. 2007, $100,1673-1685$. 
(62) Reininger, A. J.; Heijnen, H. F. G.; Schumann, H.; Specht, H. M.; Schramm, W.; Ruggeri, Z. M. Mechanism of platelet adhesion to von Willebrand factor and microparticle formation under high shear stress. Blood 2006, 107, 3537-3545.

(63) Engelmann, B. Initiation of coagulation by tissue factor carriers in blood. Blood Cells Mol. Dis. 2006, 36, 188-190.

(64) Nakamura, S.; Imamura, T.; Okamoto, K. Tissue factor in neutrophils: yes. J. Thromb. Haemost. 2004, 2, 214-217.

(65) Fischer, M.; Sperling, C.; Tengvall, P.; Werner, C. The ability of surface characteristics of materials to trigger leukocyte tissue factor expression. Biomaterials 2010, 31, 2498-2507.

(66) Mooberry, M. J.; Key, N. S. Microparticle analysis in disorders of hemostasis and thrombosis. Cytometry A 2016, 89, 111-122.

(67) Kasthuri, R. S.; Glover, S. L.; Jonas, W.; McEachron, T.; Pawlinski, R.; Arepally, G. M.; Key, N. S.; Mackman, N. PF4/heparin-antibody complex induces monocyte tissue factor expression and release of tissue factor positive microparticles by activation of FcgammaRI. Blood 2012, 119, 5285-5293.

(68) Kourtzelis, I.; Markiewski, M. M.; Doumas, M.; Rafail, S.; Kambas, K.; Mitroulis, I.; Panagoutsos, S.; Passadakis, P.; Vargemezis, V.; Magotti, P.; Qu, H.; Mollnes, T. E.; Ritis, K.; Lambris, J. D. Complement anaphylatoxin C5a contributes to hemodialysis-associated thrombosis. Blood 2010, 116, 631-639.

(69) Chu, A. J.; Beydoun, S.; Mathews, S. T.; Hoang, J. Novel anticoagulant polyethylenimine: inhibition of thrombin-catalyzed fibrin formation. Arch. Biochem. Biophys. 2003, 415, 101-108.

(70) Zhong, D.; Jiao, Y.; Zhang, Y.; Zhang, W.; Li, N.; Zuo, Q.; Wang, Q.; Xue, W.; Liu, Z. Effects of the gene carrier polyethyleneimines on structure and function of blood components. Biomaterials 2013, 34, 294-305.

(71) Tenzer, S.; Docter, D.; Rosfa, S.; Wlodarski, A.; Kuharev, J.; Rekik, A.; Knauer, S. K.; Bantz, C.; Nawroth, T.; Bier, C.; Sirirattanapan, J.; Mann, W.; Treuel, L.; Zellner, R.; Maskos, M.; Schild, H.; Stauber, R. H. Nanoparticle size is a critical physicochemical determinant of the human blood plasma corona: a comprehensive quantitative proteomic analysis. ACS Nano 2011, 5, 7155-7167.

(72) Sanfins, E.; Augustsson, C.; Dahlback, B.; Linse, S.; Cedervall, T. Size-dependent effects of nanoparticles on enzymes in the blood coagulation cascade. Nano Lett. 2014, 14, 4736-4744.

(73) Brash, J. L.; Scott, C. F.; Hove, P. t.; Wojciechowski, P.; Colman, R. W. Mechanism of transient adsorption of fibrinogen from plasma to solid surfaces: Role of the contact and fibrinolytic systems. Blood 1988, 71, 932-939.

(74) Hsu, B. R. S.; Fu, S. H.; Tsai, J. S.; Huang, Y. Y.; Huang, H. S.; Chang, K. S. S. The plasminogenplasmin fibrinolytic system accelerates degradation of alginate-poly-l-lysine-alginate microcapsules in vitro. Transplant. Proc. 1997, 29, 1877-1880.

(75) Lamba, N. C. S., Interaction of blood with artificial surfaces. In Hemostasis and thrombosis, Colman, R. M., JV; Clowes AW, George, JN; Goldhaber SZ, Ed. Lippincott William \& Wilkins: 2006. (76) Choi-Miura, N. H.; Tobe, T.; Sumiya, J.; Nakano, Y.; Sano, Y.; Mazda, T.; Tomita, M. Purification and characterization of a novel hyaluronan-binding protein (PHBP) from human plasma: it has three EGF, a kringle and a serine protease domain, similar to hepatocyte growth factor activator. $J$.

Biochem. 1996, 119, 1157-1165. 